\title{
Multiscale Simulation of Electric Propulsion Effects on Active Spacecraft
}

\author{
Antoine Brunet, Pierre Sarrailh, Jean-Charles Mateo-Velez, Jean-Michel Siguier, \\ François Rogier, Jean-François Roussel and Denis Payan ${ }^{*}$
}

January 17, 2017

\begin{abstract}
The simulation of the impact of small-scale components on the electrostatic charging of a spacecraft requires the implementation of new multiscale numerical schemes. For instance, resolving the solar array interconnects is needed to accurately simulate the current collection by a satellite with electric propulsion. We here present an implementation of the nonlinear patch method in the SPIS simulation code. This method allows for local mesh refinements by embedding a local patch in a relatively coarse mesh and iteratively solving on the different meshes. The nonlinear patch method and its SPIS implementation are detailed, and numerical simulations are provided, showing the flexibility and the efficiency of the proposed method. A simulation of a complete geostationary telecom satellite with detailed solar array interconnect is proposed.
\end{abstract}

\section{Introduction}

This article presents the implementation of the nonlinear patch numerical method in the Spacecraft Plasma Interaction Software (SPIS) plasma simulation code. This modern multiscale scheme allows the SPIS software to simulate the impact of small-scale spacecraft components on the surrounding plasma.

Electrical orbit raising (EOR) has been proposed as a solution to increase payload mass while reducing the cost of geostationary (GEO) telecommunication missions. However, the use of high-power hall-effect thrusters in GEO telecommunication spacecrafts, together with the need for higher voltages on solar array generators, raise several concerns about erosion and contamination effects on the spacecraft. Modelling of the impact of solar arrays on spacecraft charging has been studied for instance in the case of the SMART-1 spacecraft [1]. More recently, higher powers have been proposed for hall effect thrusters, and the impact on charging has been studied [2]. High densities of charge exchange (CEX) ions are expected around the spacecraft, as presented in figure 1 , and the collection of these ions by the spacecraft body and its solar arrays can

${ }^{*}$ A. Brunet, P. Sarrailh, J.-C. Mateo-Velez, J.-M. Siguier, F. Rogier and J.-F. Roussel are with ONERA - The French Aerospace Laboratory, Toulouse 31000, France (email: antoine.brunet@onera.fr; pierre.sarrailh@onera.fr; jean-michel.siguier@onera.fr; jean-charles.mateo_velez@onera.fr; francois.roussel@onera.fr)

${ }^{\dagger}$ D. Payan is with Centre National d'Études Spatiales, Toulouse 31400, France (email: denis.payan@cnes.fr)

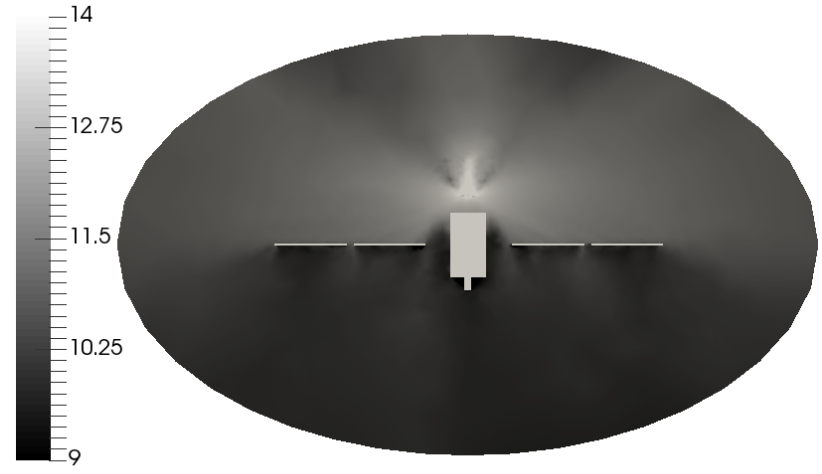

(a) CEX ions

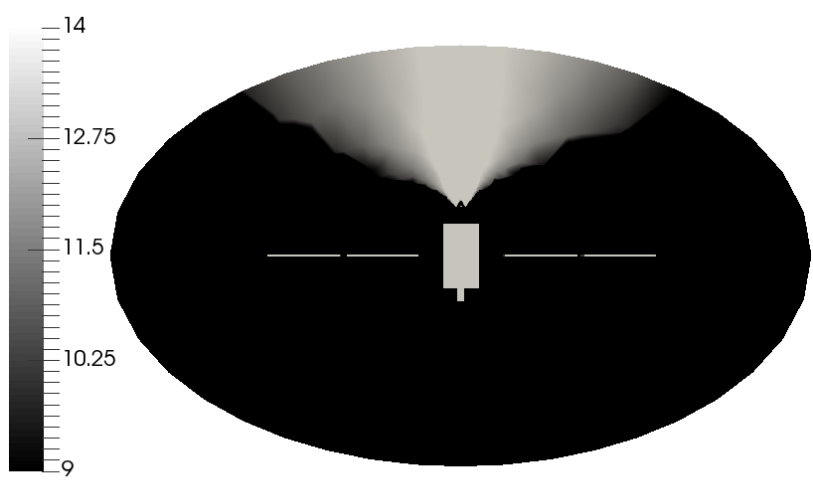

(b) Fast ions

Figure 1: Decimal logarithm of CEX and fast ion densities $\left(\mathrm{m}^{-3}\right)$

lead to serious erosion and contamination effects. Moreover, solar array interconnects are thought to have an important impact on this collection, and high voltages could lead to other effects such as snap-over, which could have an impact on the effective available power on the spacecraft.

Precise simulation of the plasma near the solar arrays are required to estimate these effects. To allow efficient and precise simulations of such small scales compared to the length of the satellite, we have implemented a modern multiscale numerical scheme called nonlinear patch method. This method allows the embedding of local refined meshes in a larger simulation domain, thus allowing the user to resolve efficiently small-scale effects in a large simulation.

We here provide a brief description of the nonlinear patch 
method and its implementation in the numerical core of the SPIS software. We then show a simple simulation of a negatively-biased interconnect in a plasma with Boltzmann electrons, compared to a reference simulation. Finally, we present the simulation of a complete geostationary telecommunication spacecraft with Hall effect thrusters and a biased solar array interconnect embedded in a patch.

\section{The Nonlinear Patch Method}

The simulation of multiscale plasma can be done with a traditional finite element method (FEM) Poisson solver, using a nonuniform mesh with characteristic sizes of elements spanning several orders of magnitude. This is the method currently used in the spacecraft Plasma Interaction Software (SPIS) to solve local sub-centimeter scales in complete satellite simulations [3]. When reaching to sub-millimeter scales, the discrepancies in the size of the elements lead to an ill-conditioned problem, which can be difficult to solve accurately. It is of interest to investigate new numerical methods, such as the patch method, which can avoid this issue by using multiple meshes to solve each scale of the simulation.

\subsection{Overview}

The patch method $[4,5]$ is a method to solve elliptic multiscale problems using multiple grids. It is a flexible domain decomposition method similar to the Chimera method used in computational fluid dynamics [6].

The algorithm used in this method is quite straightforward. The problem is successively solved on a coarse mesh and on a finer, local mesh called patch to compute corrections added to the complete solution. An extensive analysis of this method has been carried out in [4], including the derivation of convergence properties. This algorithm can be seen as a multiplicative Schwarz domain decomposition method, without conformity between the meshes. Convergence has been proven, and links between the convergence rate and the meshes geometry have been established [4] in the linear case. A more recent variant of the linear patch method has been proposed in [7] to obtain faster convergence of the method, and the nonlinear adaptation of this method also provides improved stability.

We adapted this method to solve nonlinear problems using a first order linearization scheme, as presented in [8].

\subsection{Problem Definition and Algorithm}

Consider the nonlinear Poisson-Boltzman problem

$$
-\Delta \phi+\frac{e n_{0}}{\epsilon_{0}} \exp \left(\frac{e \cdot \phi}{k T}\right)=\frac{\rho_{i}}{\epsilon_{0}}
$$

on the domain $\Omega \subset \mathbb{R}^{n}$. This equation describes the distribution of the electric potential $\phi$ in a plasma where the electrons have reach equilibirum. Here $e$ is the elementary charge, $\epsilon_{0}$ is the vacuum permittivity, $k$ is the Boltzmann constant, $n_{0}$ and $T$ are respectively the electron density at null potential and the temperature of the electrons, and $\rho_{i}$ is the charge density of the ions in the domain.

The weak formulation of equation 1 can be written

$$
\langle a(\phi) \mid \varphi\rangle=l(\varphi), \forall \varphi \in H_{0}^{1}(\Omega)
$$

Where $a$ is the Poisson-Boltzman operator, and the source term $l$ is a continuous form on $H_{0}^{1}(\Omega)$. For all $\phi \in H_{0}^{1}(\Omega)$, the Jacobian $J_{\phi}$ of $a$ near $\phi$ is continuous and coercive.

Suppose the solution $\phi$ of the problem varies rapidly in a small subdomain $\Lambda \subset \Omega$, but slowly in $\Omega \backslash \Lambda$. We define a coarse mesh of $\Omega$, with associated function space $V_{H} \subset H_{0}^{1}(\Omega)$, and a fine mesh of $\Lambda$ called patch, associated to the function space $V_{h} \subset H_{0}^{1}(\Omega)$. We set $V=V_{H}+V_{h}$ and approximate the solution $\phi$ of the continuous problem with the solution $\phi_{H h}$ of

$$
\left\langle a\left(\phi_{H h}\right) \mid \varphi\right\rangle=l(\varphi), \forall \varphi \in V
$$

It is known that this problem is not trivial to solve even when $a$ is linear, because it is generally difficult to construct a finite element basis of the function space $V$. Iterative scheme such as the patch method have been proposed to solve this problem efficiently in the linear case. We showed in [8] that it is possible to build a linearization scheme of the patch method to converge to the solution of the nonlinear problem. The resulting nonlinear patch method is similar to the Newton method used in SPIS to solve the classical monoscale case.

Let us introduce the following notation:

$\forall\left(\varphi, \phi_{\ell}, \delta \phi\right) \in V^{3},\left\langle R_{\phi_{\ell}}(\delta \phi) \mid \varphi\right\rangle=l(\varphi)-\left\langle a\left(\phi_{\ell}\right)+J_{\phi_{\ell}} \delta \phi \mid \varphi\right\rangle$

In equation $4, R_{\phi_{\ell}}(\delta \phi)$ can be seen as a residual in the linearized problem at $\phi_{\ell}$, with approximate solution $\delta \phi$. In particular, we have:

$$
R_{\phi}(\mathbf{0})=\Delta \phi-\frac{e n_{0}}{\epsilon_{0}} \exp \left(\frac{e \cdot \phi}{k T}\right)+\frac{\rho_{i}}{\epsilon_{0}}
$$

It is readily seen that equation 3 is equivalent to

$$
\left\|R_{\phi_{H h}}(\mathbf{0})\right\|=0
$$

With this notation, the proposed algorithm can be written:

1. Find $\phi_{h} \in V_{h}$ s.t. $\forall \varphi_{h} \in V_{h}$,

$$
\left\langle J_{\phi_{\ell}} \phi_{h} \mid \varphi_{h}\right\rangle=\left\langle R_{\phi_{\ell}}\left(\phi_{n}-\phi_{\ell}\right) \mid \varphi_{h}\right\rangle
$$

2. Let $\phi_{n+\frac{1}{2}}=\phi_{n} \quad+\omega \phi_{h}$

3. Find $\phi_{H} \in V_{H}$ s.t. $\forall \varphi_{H} \in V_{H}$,

$$
\left\langle J_{\phi_{\ell}} \phi_{H} \mid \varphi_{H}\right\rangle=\left\langle R_{\phi_{\ell}}\left(\phi_{n+\frac{1}{2}}-\phi_{\ell}\right) \mid \varphi_{H}\right\rangle
$$

4. Let $\phi_{n+1}=\phi_{n+\frac{1}{2}}+\omega \phi_{H}$

5. If $\left\|R_{\phi_{\ell}}\left(\phi_{n+1}-\phi_{\ell}\right)\right\|<\epsilon\left\|R_{\phi_{\ell}}(\mathbf{0})\right\|$, let $\phi_{\ell}=\phi_{n+1}$

In this algorithm, steps 1 and 3 are solving a linearized problem respectively on $V_{h}$ and $V_{H}$, steps 2 and 4 are updating the solution with a relaxation parameter $\omega \in$ $[0,2]$, and the step 5 updates the linearization when a convergence property is reached. This algorithm converges to an approximate solution to equation 3 , which is the sum of the solutions on the different meshes. 


\section{SPIS Implementation}

We here provide a summary of the implementation of the nonlinear patch method on the SPIS numerical core. SPIS is a $3 \mathrm{D}$ particle-in-cell solver using non-structured meshes for the simulation of the interaction between a spacecraft and its surrounding plasma. As with most particle-in-cell solvers, SPIS solving process consists of a Poisson solving step yielding the electrostatic potential from the charge density distribution, and a particle pushing step that propagates charges on the electric field. To avoid extensive modifications to the SPIS software, user interaction processes have been left untouched. The patch and coarse meshes are provided by the user as a unique non-connex and self-intersecting mesh. The Poisson solver and particle pusher have been modified to implement the nonlinear patch method while outputting consistent results on this unusual mesh to the other parts of the software.

SPIS uses Compressed Row Storage (CRS) to store the needed operators. This data structure has been enhanced to provide efficient partial operator application, and to efficiently compute the boundary between the patch and coarse nodes when renumbering of the nodes occurs during a Cuthill-McKee preconditioning step. These modifications allow for an efficient implementation of the patch method, in place of the classical Newton method previously implemented in SPIS.

The particle pusher has also been modified to ensure that particles entering the patch area are assigned to the correct patch cells. We use an implementation of the multimap data structure to provide constant-time localisation of a patch cell. When particles are pushed in the patch, the charge densities are deposited on both meshes, allowing to directly build the source term for the Poisson solver. The particle trajectories are always computed with the electric field of the most refined mesh.

Instead of computing the charge densities after pushing every particles, particle charges are deposited along the trajectories of the particles during the simulation. This method decreases the statistical noise inside the patch without requiring more particles, since each particle will cross more cells when in the patch than in the coarse mesh. Hence no special treatment is required when particles enter

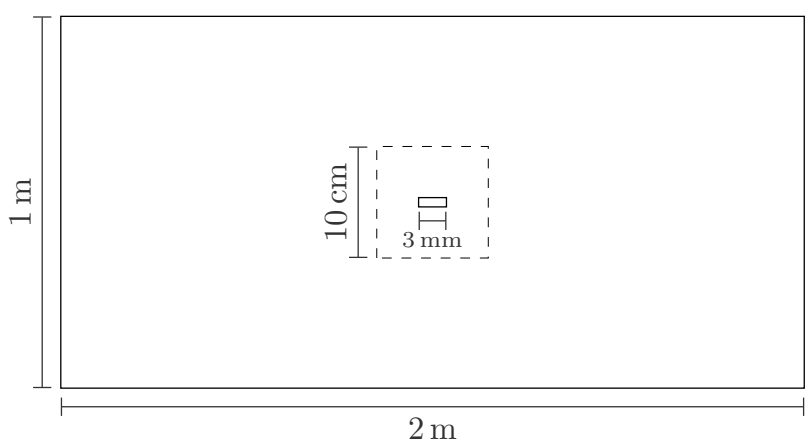

Figure 2: Spacecraft geometry (not to scale). The considered computational domain is a $50 \mathrm{~cm}$ thick box based on this geometry. The dashed squared is the patch location, the inner rectangle is the interconnect geometry.

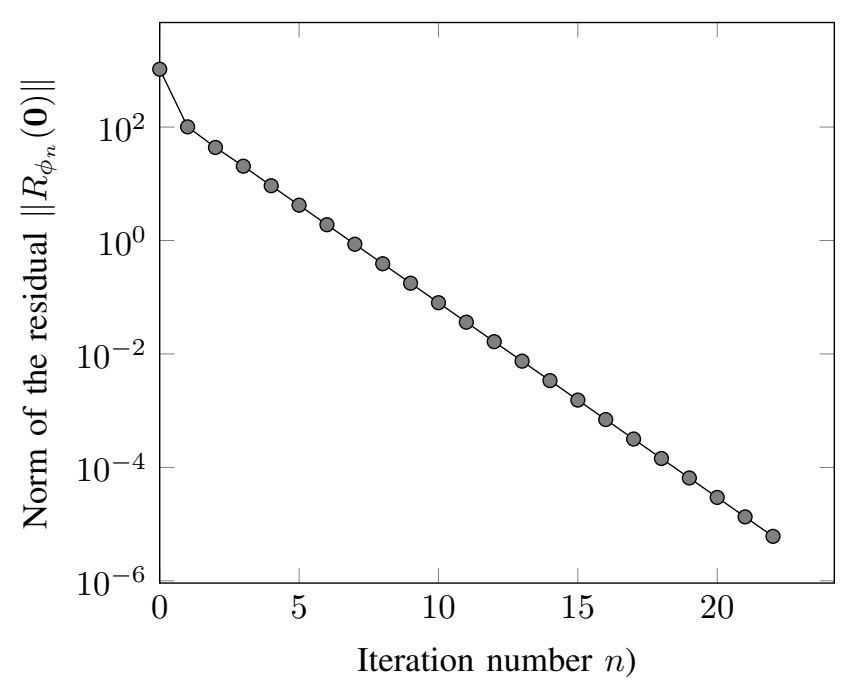

Figure 3: Convergence of the patch method for the example simulation

or leave the patch mesh.

\section{Numerical Experiments}

A theoretical analysis of the patch method and a simplified numerical example has been provided in [8]. Here we provide $3 \mathrm{D}$ numerical examples of the nonlinear patch method in SPIS. We first show a simple small interconnect embedded in a large box, validated by a detailed classical simulation. We then show a complete simulation of a geostationary telecommunication spacecraft with plasma thrusters.

\subsection{Simple Model Validation}

We here provide a simplified example of a negatively biased interconnect at $-100 \mathrm{~V}$ embedded in a relatively large computational domain. Simulations of solar array interconnects have been published several times in the literature $[9,10]$, but periodic boundary conditions on relatively small computational domains are usually used, as a model of an interconnect embedded in an infinite solar array. The simulation shown here is less representative of a real interconnect in a solar array, but uses a simplified geometry to showcase our numerical scheme.

The geometry here used is described in figure 2 . The domain is a $2 \mathrm{~m} \times 1 \mathrm{~m} \times 0.5 \mathrm{~m}$ box, and a Dirichlet boundary condition is applied on one of its largest sides. The prescribed potential on this side is computed by SPIS circuit solver dynamically between time-steps to model the charging of an ITO coated material, as described in [3]. A small $1 \mathrm{~mm} \times 3 \mathrm{~mm}$ negatively biased conductor is embedded in the center of this surface to model the interconnect. On the other sides of the domain, an homogeneous Neumann condition is applied.

The patch is automatically generated by subdivision of a selection of coarse cells, yielding around 125 refined cells per coarse cell. The obtained patch is covering a box of $10 \mathrm{~cm} \times 10 \mathrm{~cm} \times 5 \mathrm{~cm}$ around the interconnect. The typical 


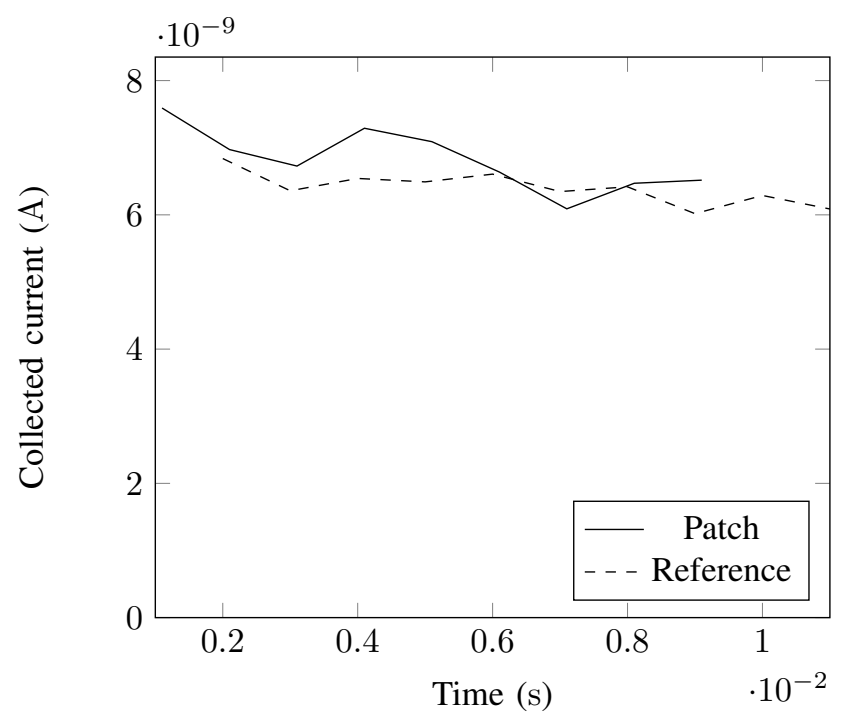

Figure 4: Total current collection by the interconnect for the reference and patch simulation

Table 1: Simulation Sizes and Integration Times

\begin{tabular}{lrrr}
\hline Simulation & Cells & Particles & Total simulation time \\
\hline Reference & 57314 & 6215277 & $695 \mathrm{~s}$ \\
Patch & 59670 & 6215100 & $639 \mathrm{~s}$ \\
\hline
\end{tabular}

size of the coarse cells range from $10 \mathrm{~cm}$ to $1 \mathrm{~mm}$, and the size of the patch cells range from $5 \mathrm{~mm}$ down to $0.2 \mathrm{~mm}$. Electrons in the plasma are modelled by a Boltzmann statistic at density $10^{12} \mathrm{~m}^{-3}$ and temperature $5 \mathrm{eV}$, and ion densities are computed by a particle-in-cell numerical model. Injected $\mathrm{Xe}+$ ions have a temperature of $1 \mathrm{eV}$, a normal drift velocity of $2 \mathrm{~km} \mathrm{~s}^{-1}$ towards the spacecraft, and a tangential velocity of $2.83 \mathrm{~km} \mathrm{~s}^{-1}$.

As shown theoretically in [8], the convergence of the method is linear, and is shown in figure 3 . This convergence is measured on the first timestep of the simulation, subsequent iterations are showing similar convergence properties, until the error reaches the roundoff errors generated by quadrature approximations.

A reference simulation has been carried out with an overrefined mesh with cells directly meshing the interconnect at $0.1 \mathrm{~mm}$, and with a large number of macro-particles to avoid statistical noise. We here give comparative results between both simulations. Table 1 presents the mesh sizes and particle counts of the reference and patch simulations, and the required integration times for these simulations. Note that given similar mesh sizes and particle counts, both methods require about the same computational time. This shows that the iterative nonlinear patch method is not more computationally expansive than the classical Newton method, while allowing the resolution of very small scales.

Figure 4 shows the collected currents on the interconnect for both simulations. A good agreement between the simulation results can be noted. The difference between the two results is well within the statistical noise of the simulation, which is around $1 \mathrm{nA}$.

Figure 5 shows the spatial distribution of the collected

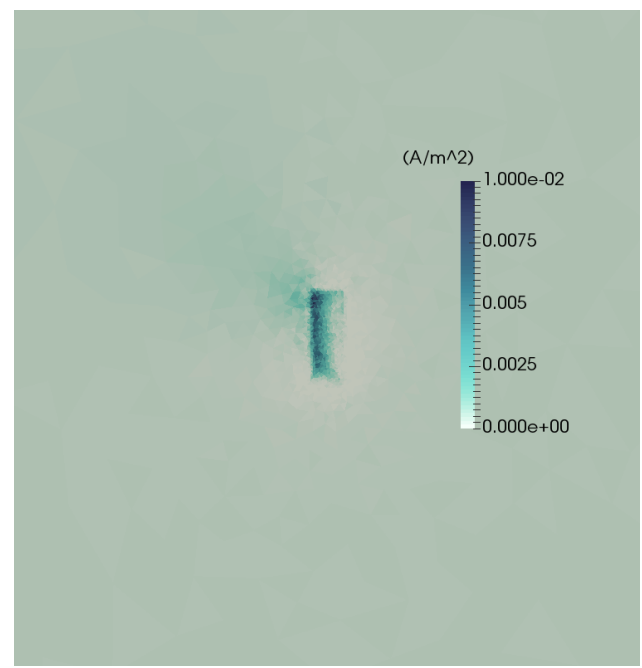

(a) Reference

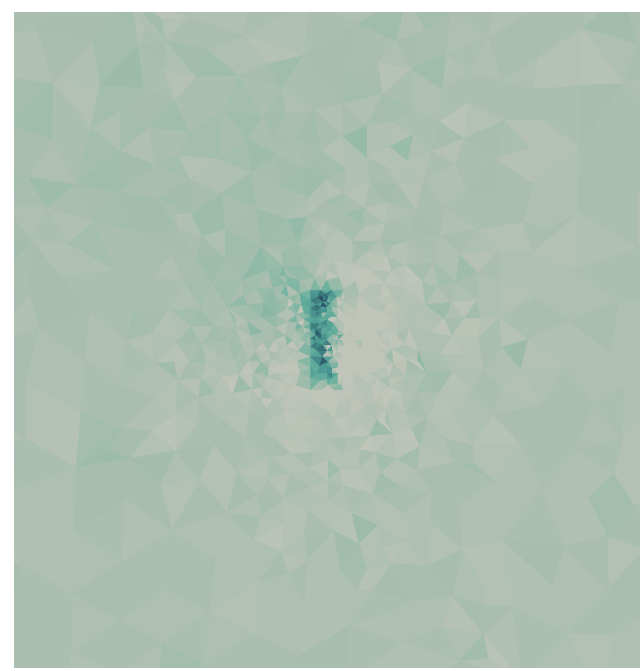

(b) Patch simulation

Figure 5: Surface current collection of ions around the interconnect at $\mathrm{t}=10 \mathrm{~ms}$. 


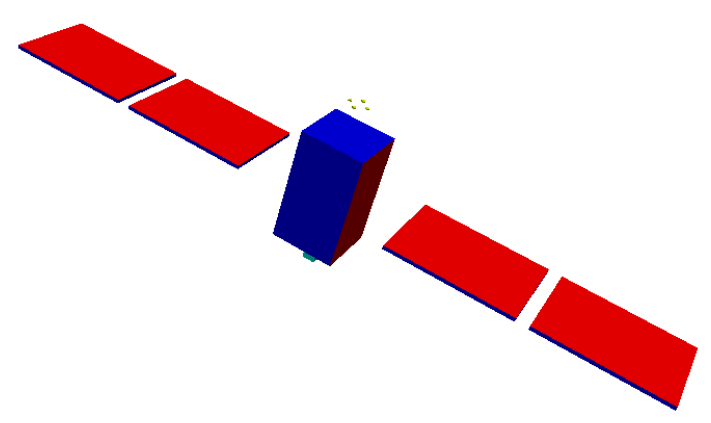

Figure 6: Spacecraft geometry. Each solar panel is $4 \mathrm{~m} \times 5 \mathrm{~m}$, a patch with a biased interconnect is embedded in one of the inner panel. The satellite spans $27 \mathrm{~m}$

ion currents around the interconnect for the reference simulation and the simulation with a patch at the same time step. Note that for the simulation with a patch, the figure shows the collection on the coarse mesh, giving a more noisy solution, which however matches the reference solution. Notice also that the collection patterns near the interconnects are complex, due to a drifting velocity of the ion population. Sufficiently refined meshes are hence needed to provide accurate results, for instance to simulate the erosion of the interconnect and of the surrounding coverglass. Multiscale numerical methods such as the nonlinear patch method are required to simulate these effects in a complete spacecraft simulation, as presented in the next section.

The numerical results presented on this example problem show that this multiscale numerical scheme is able to provide an accurate current estimation for the simulation of space plasmas down to smaller scales at an acceptable computational cost.

\subsection{Spacecraft Simulation}

Here we present an application of the nonlinear patch method for the simulation of a complete GEO spacecraft. The SPIS simulation code has previously been used to simulate the impact of high-power Hall effect thrusters on a GEO spacecraft [2]. It has been shown that satellite charging is a major factor in computing the contamination fluxes in this case. However, the direct simulation of an interconnect in a complete spacecraft is untractable using classical numerical schemes. Here we use the nonlinear patch method to simulate the current collection of a single charged interconnect.

We used the same geometry and plasma parameters as in [2], adding a local patch on one of the inner solar panels, and containing a small $1 \mathrm{~mm} \times 3 \mathrm{~mm}$ interconnect biased at $-50 \mathrm{~V}$ relatively to the spacecraft body. The spacecraft geometry is presented in figure 6 . The geometry spans $27 \mathrm{~m}$ and the body is $4.5 \mathrm{~m} \times 2.5 \mathrm{~m} \times 2.5 \mathrm{~m}$. Near the body of the spacecraft, four Hall-effect thrusters of type SPT100 are placed, and emit a total Xenon ion current of $20 \mathrm{~A}$ with an ionizing efficiency of $95 \%$, and drift energy of $160 \mathrm{eV}$. Neutrals perform charge exchange (CEX) with these fast

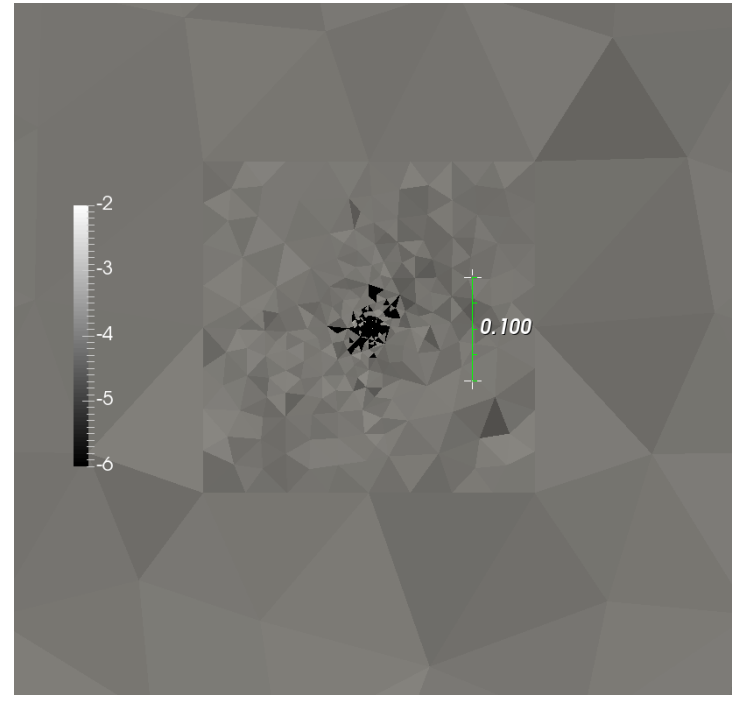

Figure 7: Decimal logarithm of surface ions collection current $\left(\mathrm{A} \mathrm{m}^{-2}\right)$ near the patch. The interconnect embedded on the solar panel locally decreases the collected currents in an area of approximately $10 \mathrm{~cm}$ diameter.

ions and generate low energy CEX ions.

The spacecraft environment is represented by a plasma density of $10^{10} \mathrm{~m}^{-3}$ and temperature of $2.8 \mathrm{eV}$. In this simulation, the spacecraft has no velocity relative to the plasma. The satellite floats at $-30 \mathrm{~V}$.

Figure 1 presents the ion densities near the spacecraft for both CEX and fast ions. Compared to results presented in [2] without the patch method, the overall charge distribution is not heavily affected by the presence of only one biased interconnect.

However, as can be seen in figure 7, a notable decrease of the collected ion current can be observed around the interconnect. As seen in the case of the previous example, current collection around the interconnect forms a complex pattern that can complicate the evaluation of the erosion and contamination of the solar arrays. The area affected by the biased interconnect has a diameter of approximately $10 \mathrm{~cm}$, which is two orders of magnitude greater than the size of the interconnect itself.

The same simulation has been carried out with a positively biased interconnect at $50 \mathrm{~V}$. To simulate the impact of all of the solar array interconnects on the spacecraft charging, we added a large $30 \mathrm{~cm} \times 30 \mathrm{~cm}$ macro-interconnect on one of the solar arrays, also positively charged at $50 \mathrm{~V}$ relatively to the spacecraft. This macro-interconnect is embedded in the coarse mesh, outside of the patch, as it would in a classical simulation.

Figure 8 shows the electric potential around the refined real-sized interconnect and around the macro-interconnect. In each case, the potential at the top of the figure is around $10 \mathrm{~V}$, due to the presence of the positively biased thrusters. In the case of the real-sized interconnect, its high potential is shielded by the surrounding negatively charged coverglass. Hence the real-sized interconnect cannot collect an important electronic current despite its relatively high potential. On the contrary, the macro-interconnect is too 


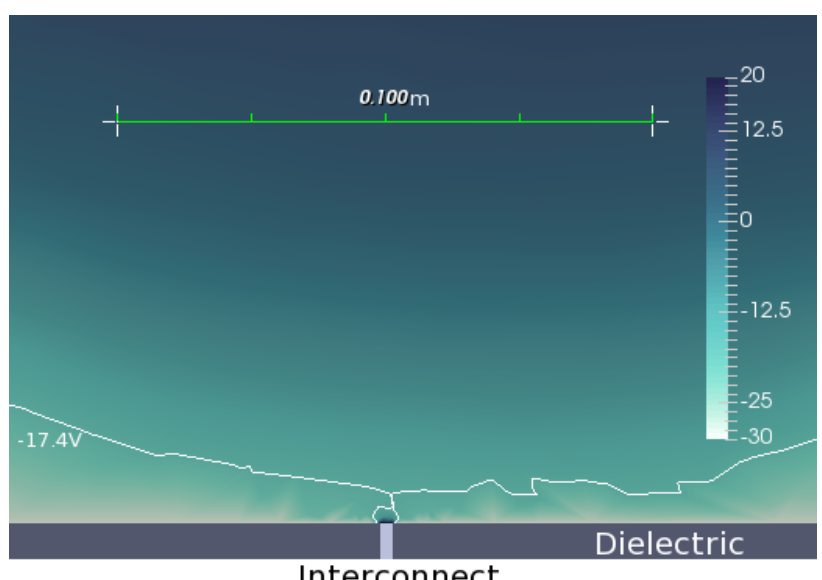

(a) Real-sized interconnect

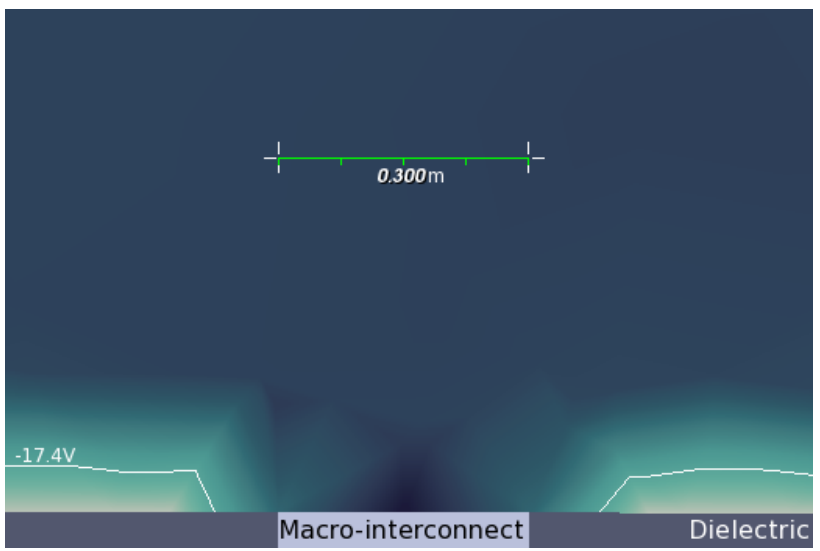

(b) Macro interconnect

Figure 8: Electric potential (V) around two types of positively biased interconnects. The spatial scale is different in the two figures to accomodate for the very different sizes of the interconnects.

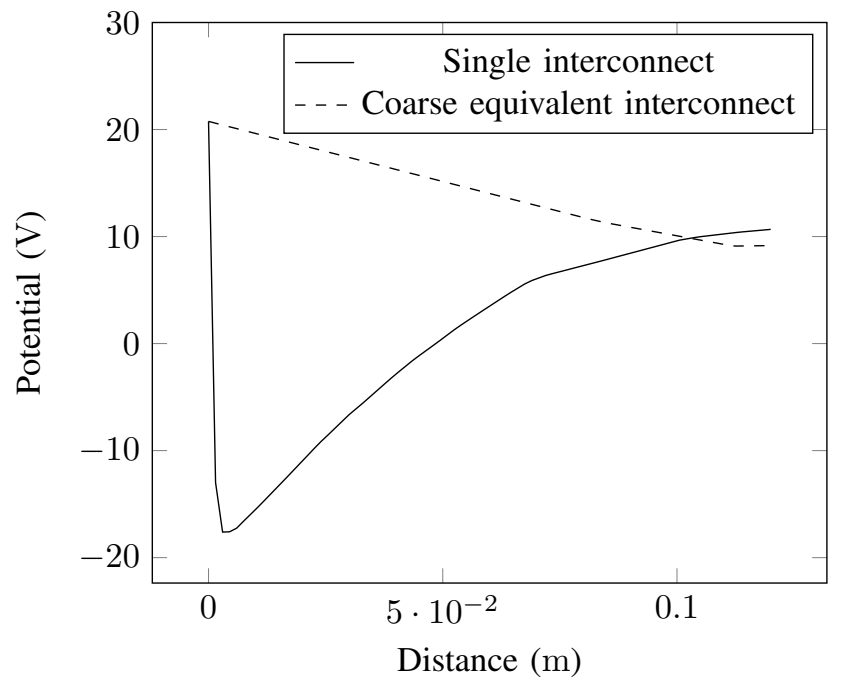

Figure 9: Electric potential (V) along the normal to a coarse interconnect with surface equivalent to the total spacecraft interconnect surface, and to a real-sized interconnect.

large to be effectively shielded by its surrounding coverglass. The current collected by individual interconnects is therefore over-estimated when using a surface-equivalent macro-interconnect.

Figure 9 shows the electric potential along the normal at the center of both interconnects. While both interconnects charge to $20.8 \mathrm{~V}$ relatively to the plasma, it can be seen that the potential around the real-sized interconnect drops rapidly to a minimum of $-17.6 \mathrm{~V}$. Since the electronic temperature is $2.8 \mathrm{eV}$, this has a major effect on the collected electronic currents, and shows that the accurate estimation of the satellite charging requires simulations resolving very small geometrical scale.

\section{Conclusion}

The nonlinear patch method allows for the simulation of the impact of small-scale spacecraft components on its environment. In particular, the implementation of this method in the SPIS simulation code, presented in this paper, can simulate solar array interconnects on a GEO spacecraft with the precision required to accurately assess the electrostatic charging of the spacecraft. Such simulations are required for the design of new missions, in particular to provide a good estimate of the erosion on the spacecraft.

It should be noted that this method cannot currently provide an effective tool to simulate a large number of smallscale elements, such as the thousands of interconnects on the surface of a solar array. It is foreseen that efficient model reduction can be used to solve this issue, as is done in other fields of numerical simulation. Such developments could bring accurate simulation of fully-detailed satellites in the space environment. 


\section{Acknowledgment}

This work is supported by the Centre National d'Études Spatiales (CNES) and Onera.

The authors would like to thank Benjamin Jeanty-Ruard from Artenum for his help in the developments involved in this paper.

\section{References}

[1] A. Hilgers, B. Thiébault, D. L. Estublier, E. Gengembre, J. A. Gonzalez del Amo, M. Capacci, J.-F. Roussel, M. Tajmar, and J. Forest, "A simple model of the effect of solar array orientation on SMART-1 floating potential," IEEE Transactions on Plasma Science, vol. 34, no. 5, Oct. 2006.

[2] J.-C. Matéo-Vélez, P. Sarrailh, S. Hess, and J.-F. Roussel, "Simulations of plasma thruster and large antenna effects on the electrostatic behavior of spacecraft in GEO," in 14th Spacecraft Charging Technology Conference, ESA/ESTEC, Noordwijk, NL, Apr. 2016.

[3] J. Roussel, F. Rogier, G. Dufour, J.-C. Mateo-Velez, J. Forest, A. Hilgers, D. Rodgers, L. Girard, and D. Payan, "SPIS Open-Source Code: Methods, Capabilities, Achievements, and Prospects," IEEE Transactions on Plasma Science, vol. 36, no. 5, pp. 2360-2368, Oct. 2008.

[4] J. Wagner, "Finite element methods with patches and applications," Ph.D. dissertation, EPF, Lausanne, Swiss Confederation, 2006.

[5] R. Glowinski, J. He, J. Rappaz, and J. Wagner, "Approximation of multi-scale elliptic problems using patches of finite elements," Comptes Rendus Mathematique, vol. 337, no. 10, pp. 679-684, Nov. 2003.

[6] J. L. Steger and J. A. Benek, "On the use of composite grid schemes in computational aerodynamics," Computer Methods in Applied Mechanics and Engineering, vol. 64, no. 1-3, pp. 301-320, Oct. 1987.

[7] J. He, A. Lozinski, and J. Rappaz, "Accelerating the method of finite element patches using approximately harmonic functions," Comptes Rendus Mathematique, vol. 345, no. 2, pp. 107-112, Jul. 2007.

[8] A. Brunet, P. Sarrailh, F. Rogier, J.-F. Roussel, and D. Payan, "Nonlinear patch method and application," in Proceedings of ECCOMAS Congress 2016, Crete Island, Greece, Jun. 2016.

[9] H. Thiemann and R. W. Schunk, "Field formation around negatively biased solar arrays in the LEO-plasma," Advances in Space Research, vol. 12, no. 12, pp. 143-146, Dec. 1992. [Online]. Available: http://www.sciencedirect.com/science/article/pii/0273117792903644

[10] _ , "Particle-in-cell simulations of sheath formation around biased interconnectors in a low-earth-orbit plasma," Journal of Spacecraft and Rockets, vol. 27, no. 5, pp. 554-562, 1990. [Online]. Available: http://dx.doi.org/10.2514/3.26179 